
\title{
Application of the Picosecond Magnetic Pulses for Inducing the Electron Motion in the Bilayer Nanowire
}

\author{
T. CHWIEJ* \\ AGH University of Science and Technology, al. A. Mickiewicza 30, 30-059 Cracow, Poland
}

\begin{abstract}
We theoretically study the effects of interaction of the single electron confined in a piece of bilayer nanowire with the picosecond magnetic pulse. It is shown that the transverse time-varying magnetic field hybridizes temporarily the vertical modes in the electron wave function which eventually stimulates its motion along the wire axis. The dynamics of such magnetically driven motion depends on the geometry of the confining potential, the effective mass of the electron and the time duration of the magnetic pulse. We show that this effect can potentially be utilized for fabrication of the magnetic valve.
\end{abstract}

DOI: 10.12693/APhysPolA.130.1217

PACS/topics: 72.25.Dc, 73.21.Hb

\section{Introduction}

The influence of the magnetic field on single electron transport in nanowire consisting of two vertically stacked layers depends, besides the strength of the field, on their mutual arrangement in space. Briefly, the static magnetic field may diminish the interlayer coupling if it is directed along the wire (longitudinal magnetic field) [1, 2], hybridize the electrons subbands in each layer separately if it is perpendicular to the wire axis and the layers [3-5], or, mix the subbands originated in different layers if it is simultaneously parallel to the layers and perpendicular to the transport axis [5]. Here, we consider the reverse of the last case, that is, we study the effect of the timevarying magnetic field on the dynamics of the electron which is initially at rest.

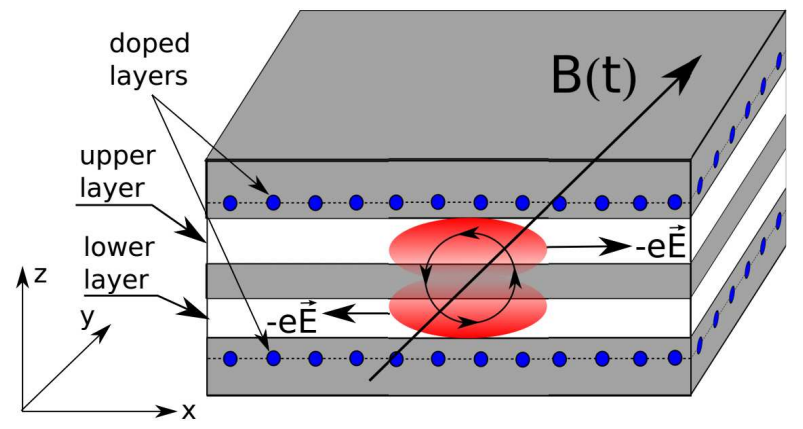

Fig. 1. Sketch of the bilayer nanowire considered in work. The temporary electric field generated by the magnetic pulse pushes the upper and lower parts of the electron density (red) in the opposite directions.

The electron is confined in the bilayer nanowire made of GaAs and InGaAs, in which two transport channels are created by two ionized $\delta$-doped layers placed near

*e-mail: chwiej@fis.agh.edu.pl its upper and lower edges (see Fig. 1). We use a simple theoretical model to show that the picosecond magnetic pulse $[6,7]$ hybridizes the vertical modes in the electron wave function which in consequence must change its group velocity. That results directly from the Faraday law $\partial \boldsymbol{B} / \partial t=-\nabla \times \boldsymbol{E}$ which predicts the formation of the temporary electric field. However, due to its rotational character, the densities confined in the upper and lower layers shall move in the opposite directions. This picture is not true for the asymmetric confinement though. Then, the majority of electron density confined in the deeper layer may drag the minority part leading to synchronization of their motions. We show that such magnetically stimulated motion of electron in nanowire depends on some factors, such as: (i) the degree of the confinement asymmetry, (ii) the effective mass of the electron, and (iii) the time duration of the magnetic pulse. We also show how this effect combined with the mechanism of the Coulomb blockade can be utilized for fabrication of simple magnetic valve.

\section{Theoretical model}

We start our considerations with a single electron Hamiltonian $\widehat{h}=(\widehat{\boldsymbol{p}}+e \boldsymbol{A})^{2} / 2 / m^{*}+V_{\mathrm{c}}$, where $e$ is a modulus of the electron charge, $\widehat{\boldsymbol{p}}$ is a momentum operator, $V_{\mathrm{c}}$ is the confining potential and $\boldsymbol{A}$ is the vector potential. We use a non-symmetric gauge for vector potential $\boldsymbol{A}=[z B(t), 0,0]$ which gives the magnetic field directed along $y$-axis $\boldsymbol{B}=[0, B(t), 0]$. In our model, the electron moves along $x$-axis in the nanowire while the spatial extent of its wave function in $y-z$ plane is limited to the square quantum well defined by $y \in[-a, a]$ and $z \in[-b, b]$. The part of $\widehat{h}$, which depends on $y$ variable, separates from the rest, and as it does not influence the motion in $x$ and $z$ directions it is skipped in further considerations. The confining potential in nanowire is assumed in separable form, i.e., $V_{\mathrm{c}}(x, z)=V_{x}(x)+V_{z}(z)$ while the surrounding barrier is assumed to be infinite. The confining potential of a square quantum well in the growth direction is modified by the ionized donors which 
are localized in the upper and lower $\delta$-doped layers [8]. Sketch of the nanostructure is shown in Fig. 1. We model the confining potential $V_{z}(z)$ with $[9] V_{z}(z)=$ $V_{m}\{\sin [\gamma(z)]+\alpha \sin [2 \gamma(z)]\}$, where $V_{m}=-150 \mathrm{meV}$, $\gamma(z)=(1+z / b) \pi / 2$ and $b=15 \mathrm{~nm}$ is the half width of quantum well in the growth direction. It simply describes the upper and lower transport layers separated by a central barrier. For $\alpha=0$ the confining potential $V_{z}$ is symmetric, while for $\alpha>0$ the upper layer is deeper than the lower one and thus may confine the major part of the electron density. Next, we diagonalize the Hamiltonian $\widehat{h}_{z}=\widehat{p}_{z}^{2} / 2 / m^{*}+V_{z}(z)$ and afterwards use its eigenfunctions $\phi_{i}(z)$ to calculate the matrix elements of Hamiltonian $\widehat{h}$, i.e., $H_{k, m}=\left\langle\phi_{k}(z)|\widehat{h}| \phi_{m}(z)\right\rangle$. These elements have the following form:

$$
\begin{gathered}
H_{k, m}=\left(\frac{\widehat{p}_{x}^{2}}{2 m^{*}}+V_{x}(x)+E_{k}\right) \delta_{k, m} \\
+\hbar \omega_{\mathrm{c}} \zeta_{k, m}^{(1)} \widehat{k}_{x}+\frac{m^{*} \omega_{\mathrm{c}}^{2}}{2} \zeta_{k, m}^{(2)},
\end{gathered}
$$

where $E_{k}$ are the eigenenergies of $\widehat{h}_{z}, \zeta_{k, m}^{(l)}=\left\langle\phi_{k}\left|z^{l}\right| \phi_{m}\right\rangle$, $\widehat{k}_{x}=\widehat{p}_{x} / \hbar=-\mathrm{i} \partial_{x}$. The cyclotron frequency $\omega_{\mathrm{c}}=$ $e B(t) / m^{*}$ is the function of time. Therefore, the second term in Eq. (1) shall change the electron momentum when the magnetic pulse is piercing the nanostructure. We define the magnetic pulse with a simple sinusoidal shape $B(t)=B_{m} \sin \left(\pi t / t_{t_{\mathrm{imp}}}\right) \Theta(t) \Theta\left(t_{\mathrm{imp}}-t\right)$, where $\Theta(t)$ is the Heaviside step function, $t_{\mathrm{imp}}$ is the time duration of the pulse and $B_{m}$ its amplitude. In calculations we used $B_{m}=5 \mathrm{~T}$ and $t_{\mathrm{imp}}=5-50 \mathrm{ps}$. The spatial part of the single electron wave function can be defined now as $\Psi(x, z, t)=\sum_{m=1}^{m} \psi_{m}(x, t) \phi_{m}(z)$. Hence, all the time dependency is put to the wave functions $\psi_{m}(x, t)$ which evolve in time according to the Schrödinger equation

$$
\mathrm{i} \hbar \frac{\partial \psi_{k}(x, t)}{\partial t}=\sum_{m=1}^{m} H_{k, m} \psi_{m}(x, t), k=1,2, \ldots, M .
$$

We found numerically the solutions of Eq. (2) for the time-varying magnetic field for time step $\Delta t=10^{-4} \mathrm{ps}$. The initial wave function $\Psi(x, z, 0)$ was determined during the diagonalization process of the Hamiltonian matrix $\boldsymbol{h}=\left[H_{k, m}\right]$ on the spatial mesh for $x$ direction. In $\boldsymbol{h}$ we have imposed the confining potential $V_{x}(x)=m^{*} \omega_{0}^{2} x^{2} / 2$ with $\hbar \omega_{0}=0.5 \mathrm{meV}$, which limits the spatial range of the electron in the nanowire. The length of the nanowire was $800 \mathrm{~nm}$ while the distance between neighboring nodes was $\Delta x=2 \mathrm{~nm}$. The $\phi_{m}(z)$ were determined in the same way but for $\Delta z=0.15 \mathrm{~nm}$. Below we study the dynamics of the electron density subjected to an action of magnetic pulse by analyzing the densities confined in both layers $\rho_{u / l}=\langle\Psi \mid \Psi\rangle_{z>0 / z<0}$, where indices $u$ and $l$ stand for the upper and the lower layer, respectively, and, the normalized expectation value of the electron position is $x_{u}=\langle\Psi|x| \Psi\rangle_{z>0} / \rho_{u}(z<0$ for $l)$.

\section{Results and discussion}

Figure 2 shows the example results of calculations for GaAs bilayer nanowire. For $\alpha=0$ (Fig. 2a) the confinement in the vertical direction is symmetric. Both layers confine thus the same amounts of the electron density. As the local electric fields generated in the layers have opposite directions, the upper and lower densities are forced to move in the opposite directions, too. Hence, the expectation value of the electron position in the nanowire does not change $\left(\alpha=0 \Longleftrightarrow\langle x\rangle=x_{u}+x_{l}\right)$. For $\alpha>0$ the dynamics of the electron density will significantly change. Then, the upper and lower electron positions, $x_{u}$ and $x_{l}$, synchronize themselves (Fig. 2c). In this case, the major part of the single electron density is localized in the upper layer. When the magnetic field starts to grow in time, the density confined in the upper layer is pushed to the right by the local electric field. Now however, it also drags the minor part of the density but against the electric field generated in the lower layer. This synchronized motion of the upper and lower densities is very stable and persists even for longer times when the magnetic pulse is finished. Interestingly, despite of such synchronous motion, the density may also oscillate between layers (Fig. 2d). It is due to a small admixture of $\phi_{2}(z)$ state in $\Psi(x, z, t)[10]$. The magnitude of $\phi_{2}(z)$ contribution to $\Psi(x, z, t)$ depends strictly on the energy splitting between the two lowest eigenstates of $\widehat{h}_{z}$. Because the difference of $E_{2}$ and $E_{1}$ is smaller for GaAs than for InGaAs due to heavier effective mass $\left(m_{\mathrm{GaAs}}^{*}=0.067\right.$ versus $\left.m_{\mathrm{InGaAs}}^{*}=0.04\right)$, the interlayer density oscillations will be always more pronounced in the nanowire made of GaAs rather than in InGaAs (not shown here). Besides the asymmetry factor, also the time duration of the magnetic pulse influences the energy transferred to the wire (Fig. 2b). For GaAs nanowire, the significant energy transfer shall be expected for short magnetic pulses with $t_{\mathrm{imp}}<10 \mathrm{ps}$ and for moderate value of the asymmetry factor, that is for $\alpha>0.01$.
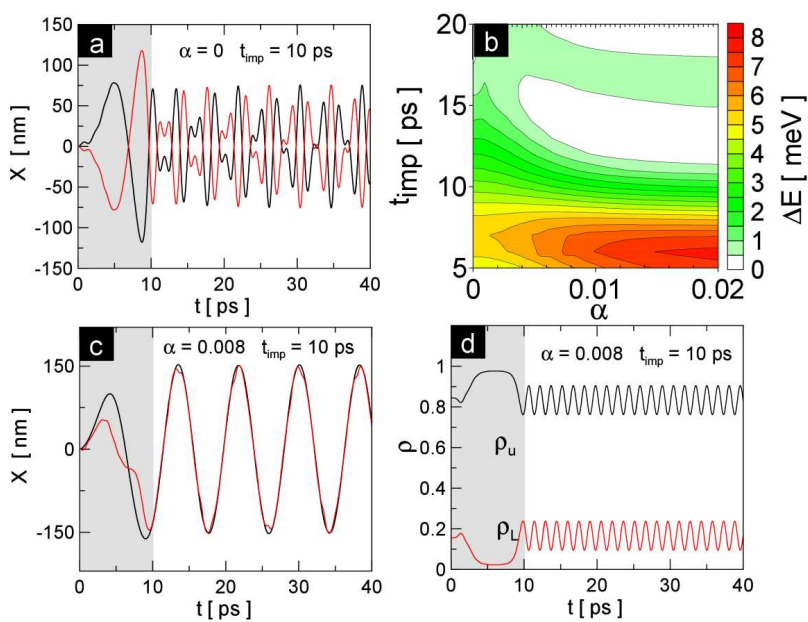

Fig. 2. Results for GaAs bilayer nanowire: (a) and (c) show the expectation value of the electron position in the upper (black) and lower (red) layers for $\alpha=0$ and $\alpha=0.008\left(t_{\mathrm{imp}}=10 \mathrm{ps}\right)$, (d) presents the interlayer density oscillations in the latter case, while (b) shows the total energy gained by the electron in dependence on asymmetry factor $\alpha$ and the time duration of the magnetic pulse. 
Despite the qualitative and quantitative differences in the results obtained for nanowires with different effective masses, we may conclude that the energy delivered to the system by the magnetic pulse can be effectively converted into the motion energy which in turn forces the electron to change its position in the bilayer nanostructure.
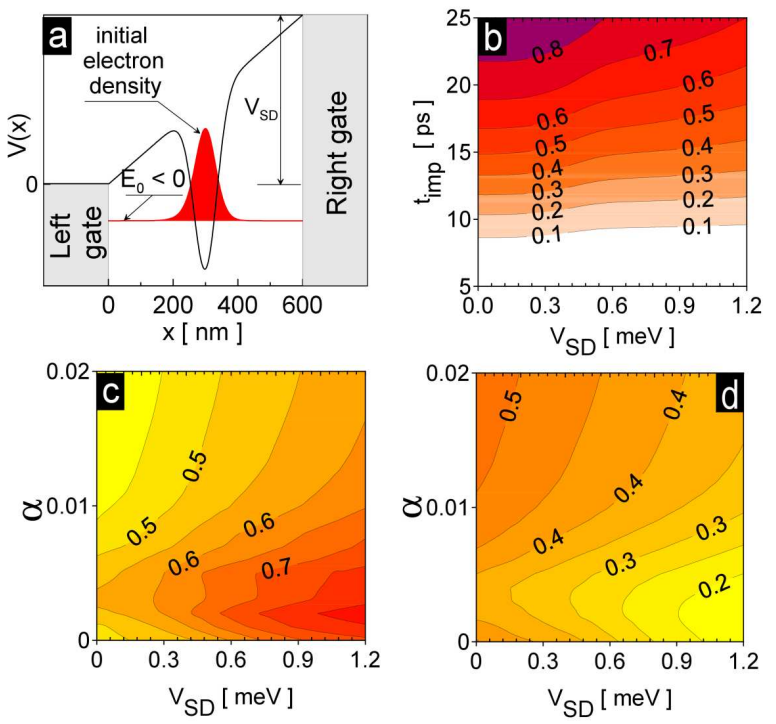

Fig. 3. (a) Quantum well (QW) placed between two gates operates in Coulomb blockade regime. After the magnetic pulse is finished a fraction of the single electron density remains in QW (b), while the rest is transferred to the left (drain) gate (c) and to the right (source) gate (d). The common parameters in (b),(c) and (d) are $m^{*}=0.067$ and $V_{0}=-1.2 \mathrm{meV}$. In (b) we used $\alpha=0.015$ while in (c) and (d) we fixed the length of magnetic pulse $t_{\mathrm{imp}}=8 \mathrm{ps}$.

We think that the effect of temporary hybridization of vertical modes, which is stimulated by the picosecond magnetic pulse, can be utilized for fabrication of the magnetic valve. The heart of such nanodevice would be the bilayer quantum well placed between two metallic gates. If the chemical potential of such single charged well lies below the chemical potentials of the gates, then the nanodevice will fully operate in the Coulomb blockade regime (see Fig. 3a), that is, the electric current will not flow in a steady state. The magnetic pulse might then excite the electron forcing it to leave the well. After its absorption in one of the reservoirs another electron might enter the empty well which again shall stop the charge flow in the system. In Fig. 3 we present the example results for such magnetically triggered nanodevice. There are shown the amount of charge remaining in the well (Fig. 3b) after the pulse is finished $\left(t=100 \mathrm{ps}>t_{\mathrm{imp}}\right)$ for $\alpha=0.015$, while (c) and (d) show how much of the single electron density is captured in the left gate (Fig. 3c) and in the right gate (Fig. 3d) in dependence of $\alpha$ and the bias voltages $V_{\mathrm{SD}}$ applied to the gates for $t_{\mathrm{imp}}=8 \mathrm{ps}$. For the confining potential of quantum well we used

$$
V_{x}(x)=V_{0} \exp \left(-\frac{\left(x-x_{0}\right)^{2}}{\sigma_{0}^{2}}\right)+e x F,
$$

where $\sigma_{0}=50 \mathrm{~nm}, V_{0}=1.2 \mathrm{meV}$ and $F$ is the electric field generated by the gates. The magnetic triggering of the charge flow in the bilayer nanosystem would be the most effective for the pulses of time duration below $t_{\text {imp }}<8$ ps. In such case, the quantum well confines less than $10 \%$ of the initial charge while the rest $90 \%$ is shifted to the left and right gates with comparable probabilities, provided that the asymmetry is meaningful. For longer pulses the amount of energy transferred to the quantum well is insufficient to make it empty (upper part of Fig. 3b), and therefore, the effectiveness of the magnetic triggering would be significantly decreased but still possible.

\section{Acknowledgments}

The work was financed by Polish Ministry of Science and Higher Education (MNiSW).

\section{References}

[1] L.G. Mourokh, A.Yu. Smirnov, S.F. Fischer, Appl. Phys. Lett. 90, 132108 (2007).

[2] S.F. Fischer, G. Apetrii, U. Kunze, D. Schuh, G. Abstreiter, Phys. Rev. B 71, 195330 (2005).

[3] J.-R. Shi, B.-Y. Gu, Phys. Rev. B 55, 9941 (1997).

[4] S.F. Fischer, G. Apetrii, U. Kunze, D. Schuh, G. Abstreiter, Nat. Phys. 2, 91 (2006).

[5] T. Chwiej, Physica B 499, 76 (2016).

[6] C. Vicario, C. Ruchert, F. Ardana-Lamas, P.M. Derlet, B. Tudu, J. Luning, C.P. Hauri, Nat. Photon. 7, 720 (2013).

[7] D.H. Auston, Appl. Phys. Lett. 26, 101 (1975).

[8] T. Chwiej, Physica E 77, 169 (2016).

[9] S.F. Fischer, G. Apetrii, U. Kunze, D. Schuh, G. Abstreiter, Phys. Rev. B 74, 115324 (2006).

[10] T. Chwiej, Phys. Rev. B 93, 235405 (2016). 